\title{
Discovery of Carbon Radio Recombination Lines in M82
}

\author{
Leah K. Morabito ${ }^{1}$, J. B. R. Oonk ${ }^{1,2}$, Francisco Salgado ${ }^{1}$, \\ M. Carmen Toribio ${ }^{2}$, Xander Tielens ${ }^{1}$ and Huub Röttgering ${ }^{1}$ \\ ${ }^{1}$ Leiden Observatory, Leiden University, \\ P. O. Box 9513, 2300 RA Leiden, The Netherlands \\ email: morabito@strw. leidenuniv.nl \\ ${ }^{2}$ Netherlands Institute for Radio Astronomy (ASTRON), \\ Postbus 2, 7990 AA Dwingeloo, The Netherlands
}

\begin{abstract}
Cold, diffuse HI clouds are a key component of the interstellar medium (ISM), and play an important role in the evolution of galaxies. Carbon radio recombination lines (CRRLs) trace this ISM stage, and with the enormous sensitivity of LOFAR we have already begun to map and constrain the physical properties of this gas in our own Galaxy. Using LOFAR's low band antenna, we have observed M82 and present the first ever extragalactic detection of CRRLs. We stack 22 lines to find a 8.5-sigma detection. The line peak to continuum ratio is $\sim 0.003$, with a FWHM of $31 \mathrm{~km} \mathrm{~s}^{-1}$. The CRRL feature is consistent with an origin in the cold, neutral medium in the direction of the nucleus of M82.
\end{abstract}

Keywords. galaxies: ISM

\section{Introduction}

M82 is a nearby $(3.52 \pm 0.02 \mathrm{Mpc}$; Jacobs et al. 2009) nuclear starburst galaxy that is part of a larger group of galaxies, and is thought to be interacting with M81 (Yun et al. 1993). M82 is host to many phases of the interstellar medium (ISM). A concentration of atomic hydrogen in the nuclear region is observed (Yun et al. 1993), where high density gas tracers associated with star formation trace a rotating ring of molecular gas (e.g., Kepley et al. 2014). The nuclear region also contains numerous HII regions (e.g., McDonald et al. 2002; Gandhi et al. 2011) and supernova remnants (SNRs; e.g., Muxlow et al. 1994; Fenech et al. 2010). The SNRs show turnovers in their spectra at low frequencies that imply the presence of ionized gas (Wills et al. 1997). The detailed interaction of these phases of the ISM is not yet understood. Carbon radio recombination lines can help by probing the cold, diffuse medium in M82.

When free electrons recombine with ions, recombination lines are produced. When the quantum numbers of the transitions are high enough $(n \gtrsim 50)$, the recombination lines appear in the radio regime and are called radio recombination lines (RRLs). These RRLs come in two different "flavours": classical and diffuse. Classical RRLs are those observed above $1 \mathrm{GHz}$, where they trace densities of about $100 \mathrm{~cm}^{-3}$ and temperature $T_{e} \sim 10^{4} \mathrm{~K}$ gas, such as that associated with HII regions. These RRLs have been in our Galaxy as well as a handful of bright nearby starbursting galaxies, including M82 (e.g., Shaver et al. 1977; Rodriguez-Rico et al. 2004).

Below $1 \mathrm{GHz}$, we observe diffuse RRLs. These trace a different phase of the ISM, with a lower density $\left(n_{e} \sim 0.01-1.0 \mathrm{~cm}^{-3}\right)$ and temperature $\left(T_{e} \sim 100 \mathrm{~K}\right)$. These lines are seen only from carbon, which has an ionization potential of $11.3 \mathrm{eV}$, lower than that of hydrogen. Not much is yet known about diffuse RRLs, but LOFAR is beginning a 
survey that over the course of the next several years will map out the Galactic CRRL absorption/emission on large (degrees) to small (3-10 arcsec) scales.

\section{Modelling CRRLs}

The observed properties of CRRLs, line width and integrated optical depth, depend sensitively on the temperature and density of the gas. Figure 1 shows the dependence of integrated optical depth on quantum number (frequency) for two different gas phases. It is clear that measurements over a range of frequencies will place tighter constraints on the gas properties.

Low frequency RRLs are not in local thermodynamic equilibrium (LTE) and we use detailed non-LTE models (Salgado et al., in prep.; also e.g., Walmsley \& Watson 1982; Shaver 1975) to calculate the departure coefficients $\left(b_{n}\right)$, which describe the departure from LTE. These departure coefficients depend on the input electron temperature, $T_{e}$, and density, $n_{e}$. When combined with an emission measure, $\mathrm{EM}_{\mathrm{CII}} \sim n_{e}^{2} L \mathrm{pc} \mathrm{cm}{ }^{-6}$, the departure coefficients can be translated directly into integrated optical depths. Singly ionised carbon has an additional dielectronic-like recombination process that must be included in the models, and results in large integrated optical depths at low frequencies. This drives the need for sensitive low

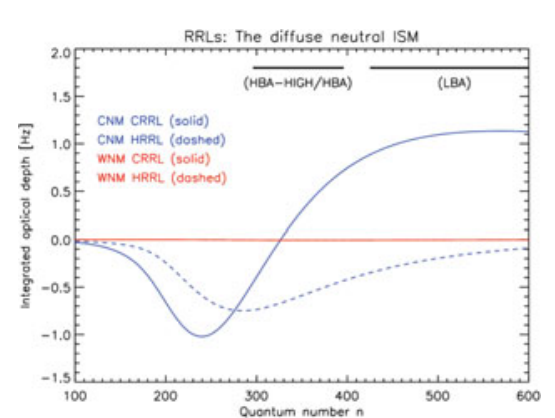

Figure 1. Integrated optical depth vs. quantum number (frequency). Cold neutral medium $(\mathrm{CNM})$ and warm neutral medium (WNM) models (Salgado et al., in prep.). The frequency ranges for LOFAR's low band antenna (LBA; $10-90 \mathrm{MHz}$ ) and high band antenna (HBA; 110-250) are marked. Models for both carbon RRLs and hydrogen RRLs are shown.

frequency instruments with enough spectral and spatial resolution to resolve the lines and localize them to a particular source.

\section{The Low Frequency Array}

The Low Frequency Array (LOFAR) consists of 24 core and 16 remote stations in the Netherlands, and 8 international stations spread across Europe. The low band antenna (LBA) covers frequencies of $10-80 \mathrm{MHz}$, while the high band antenna (HBA) covers frequencies of 120-240 MHz. The wide instantaneous bandwidth of $96 \mathrm{MHz}$ can be flexibly split to form multiple beams using LOFAR's unique phased array capabilities. Within a single observation LOFAR can therefore observe tens to hundreds of CRRL transitions. We can thus measure the dependence of the CRRL line properties as a function of frequency, to derive the physical properties of the associated gas by matching these with our models. Where necessary it also possible to stack individual line transitions in order to achieve a detection (e.g., Oonk et al. 2014).

\section{Extragalactic CRRLs}

Due to the stimulated nature of CRRLs (Shaver 1978), the line intensities of these absorption features are independent of redshift, and we will be able to probe the cold neutral medium $(\mathrm{CNM})$ out to redshifts $\gtrsim 5$. From our RRL models (Salgado et al., in prep.) we expect RRL peak optical depths of about $10^{-3}$ for typical HI column densities of $10^{21} \mathrm{~cm}^{-2}$. LOFAR is currently surveying the Northern hemisphere down to depths of $\sim 100 \mu \mathrm{Jy}$ at $\sim 150 \mathrm{MHz}$, which will yield a few hundred radio galaxies bright enough 
for CRRL searches. Although this sample will be dominated by FRI and FRII galaxies, there will still be many of the nearby well-studied starforming galaxies like M82.

The sensitivity, large field of view, frequency resolution and coverage of the Square Kilometre Array (SKA) will revolutionize the study of low-frequency recombination lines. The increased sensitivity compared to LOFAR will expand the number of extragalactic sources searchable for RRLs by almost 3 orders of magnitude, including a few hundred nearby starforming galaxies. The sensitivity of the SKA1-LOW will be good enough that we will be able to spatially resolve the brightest 25 percent of these nearby galaxies.

LOFAR is already capable of detecting CRRLs in extragalactic sources (Morabito et al. 2014, submitted), and we have begun a pilot survey for extragalactic CRRLs which will set the framework on which to base our future SKA studies.

\section{Observations and Data Reduction}

We used LOFARs LBA to observe M82 in the frequency range of $30-78 \mathrm{MHz}$ (observation details given in the table to the right). The calibrator was processed using the radio observatory's calibrator pipeline, and the amplitude solutions were transferred to the target. We imaged each channel separately for a total of 69 subbands, deliberately chosing low-resolution imaging parameters to speed up the processing time. We used only

\begin{tabular}{|l|l|}
\hline Project ID & LC0_043 \\
RA (J2000) & 09h55m52.7s \\
DEC (J2000) & $+69 d 40 \mathrm{~m} 46 \mathrm{~s}$ \\
Observing date & 2013 Feb 21 \\
On-source time & $5.0 \mathrm{~h}$ \\
Frequency range & $30-78 \mathrm{MHz}$ \\
No. SB & 244 \\
SB width & $0.1953 \mathrm{MHz}$ \\
Channels per SB & 128 \\
Velocity res. & $6-16 \mathrm{~km} \mathrm{~s}^{-1}$ \\
Simultaneous Cal. & $3 \mathrm{C} 196$ \\
\hline
\end{tabular}

the 24 core stations to make the images. This provided resolutions of $\sim 200 \times 300$ arcsec. The images were smoothed to $400 \times 400$ arcsec. We rejected five subbands that were excessively noisy, and extracted the spectra from elliptical regions in each subband.

There were 22 subbands with $\alpha$-transition $(\Delta n=1)$ CRRLs within $48-64 \mathrm{MHz}$. We trimmed noisy edge channels and fit the continuum in each subband with a low (first or second) order polynomial, after blanking the expected line locations. The subbands were each divided by their continuum fit, and we subtracted unity to place the continuum at zero.

The CRRLs are not detected individually, so we stack the 22 transitions together. Before doing this, we must know what radial velocity to use to predict the rest-frame line centres for stacking. To determine the radial velocity we cross-correlated a template spectrum of CRRLs (sampled it in the same way as the observed spectrum) and the observed spectrum. We repeat this process for a range of redshifts, and find a peak at $v=219 \mathrm{~km} \mathrm{~s}^{-1}(z=0.00073)$.

Using this radial velocity, we stacked the continuum-subtracted subbands containing CRRLs at the rest-frame of M82. All subbands were first converted to radial velocity, relative to the local standard of rest (LSR). The individual points make up a composite spectrum that has irregular sampling, with more dense, evenly spaced points towards the central region, roughly $\pm 200 \mathrm{~km} \mathrm{~s}^{-1}$ around the CRRL feature. After testing various smoothing methods to reduce the noise, we used a first order Savitzky-Golay filter (Savitzky \& Golay 1964) with a width of 31 data points to smooth the final spectrum (see Figure 7)

To test the validity of the detection we performed a series of checks: we used a "jackknife' procedure to rule out that the stacked spectrum could be dominated by one subband; we extracted the spectrum from the background sky in the images and stacked the same subbands in the same manner; we introduced random velocity shifts to each subband and stacked in the same manner. From these tests, we conclude that the feature is likely to be real. 


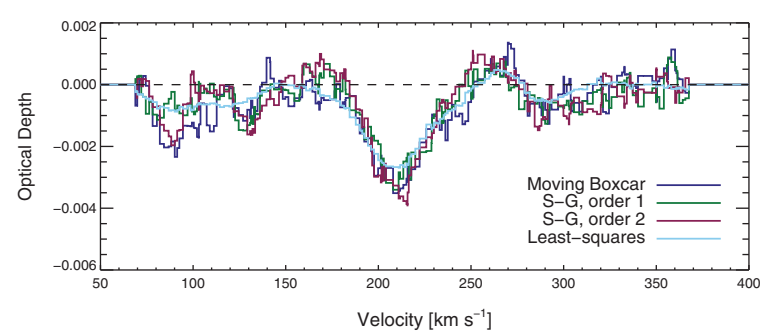

Figure 2. The stacked spectrum, smoothed in a variety of ways that each produce a similar absorption feature.

\section{Results}

The final spectrum is an average CRRL line profile for quantum levels $478 \leqslant n \leqslant 508$. We fit the CRRL feature with a Gaussian of depth $3 \times 10^{-3}$, a FWHM of $31 \mathrm{~km} \mathrm{~s}^{-1}$, a centre of $211 \mathrm{~km} \mathrm{~s}^{-1}$, and an additive offset of $-2 \times 10^{-4}$. The centroid velocity places the line within the nuclear region of M82, as inferred from other gas tracers (Kepley et al. 2014; Wills et al. 1997). A full analysis of the detected CRRL feature and its association with other gas tracers is presented in Morabito et al., 2014 (submitted).

\section{Conclusions}

We have detected, for the first time, CRRLs in an extragalactic source, M82. The CRRL line centroid indicates that gas that it traces is located in the nuclear region of M82. This single detection is not sufficient to constrain the gas temperature, density, and emission measure. Thus we are working towards detecting CRRLs at higher frequencies.

The implications of this detection are far-reaching. The intensity, and therefore observability, of these CRRL absorption lines scales only with flux, and is redshift-independent. The number of sources in which we could potentially observe CRRL absorption at low frequencies with LOFAR is many hundreds. This is the driver for a pilot survey to gather a census of extragalactic sources of CRRLs and start to constrain the evolution of the cold neutral medium over cosmological times.

\section{Acknowledgements}

LKM acknowledges financial support from NWO Top LOFAR project, project n. 614.001.006.

\section{References}

Fenech, D., Beswick, R., Muxlow, T. W. B., Pedlar, A., \& Argo, M. K. 2010, MNRAS, 408, 607 Gandhi, P., Isobe, N., Birkinshaw, M., et al. 2011, PASJ, 63, 505

Jacobs, B. A., Rizzi, L., Tully, R. B., et al. 2009, AJ, 138, 332

Kepley, A. A., Leroy, A. K., Frayer, D., et al. 2014, ApJ, 780, L13

McDonald, A. R., Muxlow, T. W. B., Wills, K. A., Pedlar, A., \& Beswick, R. J. 2002, MNRAS, 334,912

Muxlow, T. W. B., Pedlar, A., Wilkinson, P. N., et al. 1994, MNRAS, 266, 455

Oonk, J. B. R., van Weeren, R. J., Salgado, F., et al. 2014, MNRAS, 437, 3506

Rodriguez-Rico, C. A., Viallefond, F., Zhao, J.-H., Goss, W. M., \& Anantharamaiah, K. R. 2004, ApJ, 616, 783

Savitzky, A. \& Golay, M. J. E. 1964, Analytical Chemistry, 36, 1627

Shaver, P. A. 1975, Pramana, 5, 1

Shaver, P. A., Churchwell, E., \& Rots, A. H. 1977, A\&A, 55, 435

Shaver, P. A. 1978, A\&A, 68, 97

Walmsley, C. M. \& Watson, W. D. 1982, ApJ, 260, 317

Wills, K. A., Pedlar, A., Muxlow, T. W. B., \& Wilkinson, P. N. 1997, MNRAS, 291, 517

Yun, M. S., Ho, P. T. P., \& Lo, K. Y. 1993, ApJ, 411, L17 